\title{
Generalized Area Law under Multi-parameter Rotating Black Hole Spacetime
}

\author{
Masakatsu Kenmoku ${ }^{1}$, Yuki Kobayashi ${ }^{2}$ \\ Department of Physics, Nara Women's University, Nara 630-8506, Japan
}

\begin{abstract}
We study the statistical mechanics for quantum scalar fields under the multiparameter rotating black hole spacetime in arbitrary $D$ dimensions. The method of analysis is general in the sense that the metric does not depend on the explicit black hole solutions. The generalized Stefan-Boltzmann's law for the scalar field is derived by considering the allowed energy region properly. Then the generalized area law for the scalar field entropy is derived by introducing the invariant regularization parameter in the Rindler spacetime. The derived area law is applied to Kerr-AdS black holes in four and five dimensions. Thermodynamic implication is also discussed.
\end{abstract}

PACS number(s): 04.62.+v, 04.70.-s, 04.70.Dy, 97.60.Lf

\footnotetext{
${ }^{1}$ E-mail address: kenmoku@asuka.phys.nara-wu.ac.jp

${ }^{2}$ E-mail address: kobayashi@asuka.phys.nara-wu.ac.jp
} 


\section{Introduction}

Recently many evidences of rotating black holes are observed. Such black holes are well described by axisymmetric solutions of the Einstein's field equations $[1,2,3]$. Furthermore rotating black holes in higher dimensions [4] and in the (anti)- de Sitter spacetime of (negative) cosmological term have been had great interests theoretically in view of the string theory, M-theory and the AdS/CFT correspondence $[5,6,7]$.

The matter fields may be absorbed into black holes by strong gravity and the information of the matter fields may transformed to the black holes. In order to satisfies the first and second laws of thermodynamics, black hole themselves have their own entropy proportional to the area of the horizon, which is known as the black hole thermodynamics $[8,9,10,11]$. Black holes attract matter fields around them and into their horizon. On the other hand black holes may radiate some matter fields by the strong gravitational acceleration $[12,13,14]$. Therefore it is worthwhile to study the matter filed contribution to black hole thermodynamics as a quasi-equilibrium state around black holes.

The scalar field contribution to the entropy under Schwarzschild spacetime was studied extensively and the area law was shown to hold $[15,16,17,18,19]$. Because of the super-radiant stability and/or instability [20], there were some troubles to derive the area law for the scalar field under the rotating black hole spacetime in $(2+1)$ and $(3+1)$ dimensions. For Bañados-Teitelboim-Zanelli (BTZ) black hole spacetime in $(2+1)$ dimensions [21, 22, 23, 24] and Kerr black hole spacetime in $(3+1)$ dimensions [25, 26, 27], some additional divergences appeared in the statistical sum of quantum states of the scalar fields, and then the non-rotating limit cannot be taken. Another difficulty in BTZ spacetime in $(2+1)$ dimension was that the result depends on the method of calculation in addition to the above difficulties. We have studied the scalar field contribution to the rotating black hole entropy in single-parameter case in our previous paper[28]. We have taken into account the energy restriction for scalar fields in the statistical integration, which define the Boltzmann' factor well defined. We have also introduced the zenithal angle dependent regularization parameter in original spacetime, which derive the area law for the scalar field. The obtained area law of the entropy is applied to BTZ black hole spacetime and Kerr black hole spacetime, where two methods are adopted: semi-classical method and Euclidean path integral method in order to confirm the result.

In this paper, we extend the method in our previous paper [28] to multi- 
parameter rotating black hole spacetime in arbitrary $D$ dimensions. We try to improve the method to obtain the area law in the following points.

(i) Back ground black hole metric is required minimally and is in possible general form.

(ii) The energy restriction for scalar fiels will be derived ctj rigorously. This is important in performing the energy integration in statistical mechanics.

(iii) The regularization parameter will be defined systematically in the Rindler spacetime. By this definition, the regularization parameter will become invariant under radial coordinate transformation.

Under these considerations, we study the statistical mechanics for scalar fields under the multi-parameter rotating black hole spacetime by adopting the Euclidean path integral method. The obtained thermodynamic quantities of scalar fields consist of the product of temperature part and volume part in the optical space, which are in the form of generalized Stefan-Boltzmann's law. The main contribution to entropy is from the optical volume near the horizon, which leads the generalized area law of the entropy naturally. Here we introduce the regularization parameter in the Rindler spacetime to cut-off the ultra-violet divergence on the horizon. The ultra-violet cutoff for non-rotating black holes is originally introduced in the brick wall model by 't Hoot[16]. The generalized area law is applied to Kerr-(anti)-de Sitter (Kerr-AdS) black holes in four and five dimensions. Kerr-AdS spacetime is interesting in view of recent development of the AdS/CFT correspondence to understand the non-perturbative effects. Higher dimensional multi-parameter rotating black holes are also interesting to lead the deeper understanding of the black hole thermodynamics. The obtained thermodynamic quantities for scalar fields are shown to satisfy the thermodynamic (Gibbs-Duhem) relation and the first law of thermodynamics. It is interesting that they also satisfy the thermodynamics in the language of black hole variables. This implies that the thermodynamics for scalar fields can be consistently considered to be the thermodynamics for the black hole itself.

The organization of this paper is the following. In section 2, the statistical mechanics for the scalar fields is studied. The generalized Stefan-Boltzmann' law is derived in section 2.1 and the generalized area law is derived in section 2.2. In section 3 , the generalized area law is applied to Kerr-AdS black hole spacetime in $(3+1)$ and $(4+1)$ dimensions. The relation between the thermodynamics for 
scalar fields and that of the black holes is studied in section 4. Summary and discussions are given in the last section.

\section{Statistical Mechanics for Scalar Field under Multi-parameter Rotating Black Hole Space- time}

In this section, we study the statistical mechanics for the quantum scalar field under multi-parameter rotating black hole spacetime in arbitrary $D$ dimensions. Our method of analysis is general in the sense that the metric does not depend on the explicit black hole solutions. We first study the generalized StefanBoltzmann's law by adopting the Euclidean path integral method. This method leads to the optical space representation for the thermodynamic quantities naturally. To derive the generalized area law, we evaluate the scalar field entropy in the Rindler spacetime near the black hole horizon. We adopt units such that $c=\hbar=k_{\mathrm{B}}=G=1$ unless otherwise specified.

\subsection{Generalized Stefan-Boltzmann's law}

We set the $D$-dimensional polar coordinate as

$$
x^{\mu}=\left(x^{0}, x^{1}, x^{2}, \cdots, x^{D-1}\right)=\left(t, \varphi_{1}, \cdots, \varphi_{p}, \theta_{1}, \cdots, \theta_{q}, r\right),
$$

where the number of azimuthal angles $\varphi_{a}$ is $p$ and that of zenithal angles $\theta_{m}$ is $q$, where $p+q+2=D$. The line element is assumed to be of the form

$$
\begin{aligned}
d s^{2}= & \sum_{\mu, \nu=0}^{D-1} g_{\mu \nu} x^{\mu} x^{\nu} \\
= & g_{t t} d t^{2}+2 \sum_{a=1}^{p} g_{t \varphi_{a}} d t d \varphi_{a}+\sum_{i, j=1}^{D-1} h_{i j} d x^{i} x^{j} \\
= & g_{t t} d t^{2}+2 \sum_{a=1}^{p} g_{t \varphi_{a}} d t d \varphi_{a}+\sum_{a, b=1}^{p} g_{\varphi_{a} \varphi_{b}} d \varphi_{a} d \varphi_{b} \\
& +\sum_{m, n=1}^{q} g_{\theta_{m} \theta_{n}} d \theta_{m} d \theta_{n}+g_{r r} d r^{2}
\end{aligned}
$$


where special metric components $h_{i j}$ in the second line are defined as

$$
h_{i j}:=\left(g_{\varphi_{a} \varphi_{b}}, g_{\theta_{m} \theta_{n}}, g_{r r}\right),
$$

where they are already in the black diagonal form with respect to $\varphi_{a}, \theta_{m}$ and $r$. Our assumptions on the metric are the followings:

$\diamond$ Metric Condition 1 :

The off-diagonal metrices related to the time component are those between time and azimuthal components $g_{t \varphi_{a}}$ in the second terms of Eq.(2.2).

$\diamond$ Metric Condition 2:

All metrices are assumed to be functions of radial variable $r$ and zenithal angles $\theta$ and do not depend on time $t$ and azimuthal angles $\varphi_{a}$.

We use the suffix notation: $\mu, \nu=0, \cdots, D-1$ for full $D$ dimensional spacetime, $i, j=1, \cdots, D-1$ for special components $a, b=1, \cdots, p$ for azimuthal angle components $(\varphi)$ and $m, n=1, \cdots, q$ for zenithal angle components $(\theta)$ in the followings.

With the metric in Eq.(2.2), the matter action for the scalar field $\Phi$ of mass $m$ in $D$ dimension is

$$
\begin{aligned}
& I_{\text {scalar }}=\int d^{D} x \sqrt{-g} \mathcal{L}_{\text {scalar }}(x) \\
& \mathcal{L}_{\text {scalar }}(x)=-\frac{1}{2}\left(g^{\mu \nu} \partial_{\mu} \Phi(x) \partial_{\nu} \Phi(x)+m^{2} \Phi(x)^{2}\right)
\end{aligned}
$$

The canonical momentum of the scalar field is defined by

$$
\Pi(x):=\frac{\partial \mathcal{L}_{\text {scalar }}(x)}{\partial \partial_{t} \Phi(x)}=-g^{t t} \partial_{t} \Phi(x)-\sum_{a=1}^{p} g^{t \varphi_{a}} \partial_{\varphi_{a}} \Phi(x),
$$

and the quantization condition is given by

$$
\left.[\Phi(x), \Pi(y)]\right|_{t=t^{\prime}}=i \frac{\delta^{D-1}(x-y)}{\sqrt{-g}} .
$$

Because the metric components in Eq.(2.2) are assumed not to depend on $t$ and $\varphi_{a}(a=1, \cdots, p)$, there exist $(p+1)$ Killing vectors:

$$
\xi_{t}=\partial_{t}, \xi_{\varphi_{a}}=\partial_{\varphi_{a}} \quad(a=1, \cdots, p) .
$$


The existence of the Killing vectors implies the conservations of the total energy $H$ and the azimuthal angular momentum $P_{\varphi_{a}}$ of the scalar field, which are defined by

$$
\begin{aligned}
H: & =-\int_{\Sigma}\left(\xi_{t}\right)_{\mu} \mathcal{T}^{\mu t} d \Sigma_{t}=\int d^{D-1} x \sqrt{-g}\left(\Pi \partial_{t} \Phi-\mathcal{L}_{\text {scalar }}\right), \\
P_{\varphi_{a}}: & =\int_{\Sigma}\left(\xi_{\varphi_{a}}\right)_{\mu} \mathcal{T}^{\mu t} d \Sigma_{t}=\int d^{D-1} x \sqrt{-g}\left(-\Pi \partial_{\varphi_{a}} \Phi\right),
\end{aligned}
$$

where the energy-momentum tensor is $\mathcal{T}^{\mu \nu}=-(2 / \sqrt{-g})\left(\delta I_{\text {scalar }} / \delta g_{\mu \nu}\right)$. A new Killing vector $\eta$ is introduced combining ( $\mathrm{p}+1)$ Killing vectors in Eq.(2.7) linearly as

$$
\eta:=\xi_{t}+\sum_{a=1}^{p} \Omega_{\mathrm{H} a} \xi_{\varphi_{a}} .
$$

Its quadratic form satisfies the identical relation:

$$
\begin{aligned}
\eta^{2} & =g_{t t}+2 \sum_{a=1}^{p} g_{t \varphi_{a}} \Omega_{\mathrm{H} a}+\sum_{a, b=1}^{p} g_{\varphi_{a} \varphi_{b}} \Omega_{\mathrm{H} a} \Omega_{\mathrm{H} b} \\
& =\frac{1}{g^{t t}}+\sum_{a, b=1}^{p} g_{\varphi_{a} \varphi_{b}}\left(\Omega_{\mathrm{H} a}-\Omega_{a}\right)\left(\Omega_{\mathrm{H} b}-\Omega_{b}\right),
\end{aligned}
$$

where $\Omega_{a}$ and $\Omega_{\mathrm{H} a}$ are the angular velocities with respect to azimuthal angles $\varphi_{a}$ at an arbitrary position $x^{\mu}$ and at the black hole horizon $r=r_{\mathrm{H}}$ as

$$
\Omega_{a}:=\frac{g^{t \varphi_{a}}}{g^{t t}} \quad, \quad \Omega_{\mathrm{H} a}:=\left.\frac{g^{t \varphi_{a}}}{g^{t t}}\right|_{r=r_{\mathrm{H}}} \quad(a=1, \cdots, p) .
$$

The derivation of the identity equation (2.10) is given in appendix A. This equation shows that the new Killing vector $\eta$ is null and is future directed at the horizon. Corresponding to the new Killing vector $\eta$, a new conserved quantity is introduced combining the energy and the angular momenta in Eq.(2.8) as

$$
H-\sum_{a=1}^{p} \Omega_{\varphi_{a}} P_{\varphi_{a}}=-\int_{\Sigma} \eta^{\mu} T_{\mu t} d \Sigma^{t}=: \int d^{D-1} x \sqrt{-g} \mathcal{H}^{\prime},
$$

where the newly defined Hamiltonian density $\mathcal{H}^{\prime}$ is given by

$$
\begin{aligned}
\mathcal{H}^{\prime} & =\Pi \partial_{t} \Phi-\mathcal{L}_{\text {scalar }}+\sum_{a=1}^{p} \Omega_{\varphi_{a}} \Pi \partial_{\varphi_{a} \Phi} \\
& =\frac{1}{2}\left(-\frac{\Pi^{2}}{g^{t t}}+\sum_{i, j=1}^{D-1} h^{i j} \partial_{i} \Phi \partial_{j} \Phi+m^{2} \Phi^{2}\right)+\sum_{a=1}^{p}\left(\Omega_{\mathrm{H} a}-\Omega_{a}\right) \Pi \partial_{\varphi_{a}} \Phi,
\end{aligned}
$$


where $h^{i j}$ denotes the contravariant component of the special metric $h_{i j}$ in Eq.(2.3):

$$
\sum_{j=1}^{D-1} h_{i j} h^{j k}=\delta_{i}^{k} \quad \text { with } \quad h^{i j}:=\left(g^{\varphi_{a} \varphi_{b}}-\frac{g^{t \varphi_{a}} g^{t \varphi_{b}}}{g^{t t}}, g^{\theta_{m} \theta_{n}}, g^{r r}\right) .
$$

The derivation of the expression of Eq.(2.13) is given in appendix B. The expression (2.13) shows that the newly introduced energy $H-\sum_{a=1}^{p} \Omega_{H_{a}} P_{\varphi_{a}}$ is positive definite near the horizon $r \simeq r_{\mathrm{H}}$ :

$$
H-\sum_{a=1}^{p} \Omega_{H_{a}} P_{\phi_{a}}=\int d^{D-1} x \sqrt{-g} \mathcal{H}^{\prime} \geq 0 .
$$

In the following, we adopt the near horizon approximation $\Omega_{\mathrm{H} a} \simeq \Omega_{a}$ near $r \simeq r_{H}$, that is, the cross term in $\mathcal{H}^{\prime}$ in Eq.(2.13) is neglected and the energy restriction (2.15) holds.

Next we consider the partition function of the temperature $T=1 / \beta$ as

$$
Z=\operatorname{Tr}\left[\exp \left(-\beta\left(H-\sum_{a=1}^{p} \Omega_{\mathrm{H} a} P_{\varphi_{a}}\right)\right)\right] .
$$

The exponent of the Boltzmann factor $H-\sum_{a=1}^{p} \Omega_{\mathrm{H} a} P_{\varphi_{a}}$ is understood taking account of the multi-parameter rotation effect according to the Hartle-Hawking vacuum [15] and is positive definite near the horizon as shown in Eq.(2.13), which ensure the partition function to be well defined. In order to calculate the partition function, we express it in the Euclidean path integral form as

$$
Z=\int\left[\mathcal{D} \Phi \mathcal{D} \Pi g_{E}^{1 / 2}\right] \exp \left(\int_{0}^{\beta} d \tau \int d^{D-1} x \sqrt{g_{E}}\left(i \Pi \partial_{\tau} \Phi-\mathcal{H}^{\prime}\right)\right),
$$

where the Euclidean time $\tau=i t$, the Euclidean metric $g_{\tau \tau}=-g_{t t}, g_{\tau \varphi_{a}}=-i g_{t \varphi_{a}}$ and its determinant $g_{E}$ are used. The periodic boundary condition for the scalar field is required in the integration: $\Phi(x, \tau=\beta)=\Phi(x, \tau=0)$.

After performing the momentum field $\Pi$ integration and the integration by parts, the partition function becomes

$$
Z=\int\left[\mathcal{D} \Phi g_{E}^{1 / 4}\left(g^{\tau \tau}\right)^{1 / 2}\right] \exp \left(-\int_{0}^{\beta} d \tau \int d^{D-1} x, \frac{\sqrt{g_{E}} g^{\tau \tau}}{2} \Phi \bar{K} \Phi\right),
$$

where $\bar{K}$ denotes the kernel

$$
\bar{K}:=-\partial_{\tau}^{2}-\frac{1}{g^{\tau \tau}}\left(\sum_{i, j=1}^{D-1} \frac{1}{\sqrt{h}} \partial_{i}\left(\sqrt{h} h^{i j} \partial_{j}\right)-m^{2}\right)
$$


in the optical space of the metric:

$$
d \bar{s}^{2}=d \tau^{2}+g^{\tau \tau} \sum_{i, j=1}^{D-1} h_{i j} d x^{i} d x^{j} .
$$

In Eq.(2.19), the determinant of $h_{i j}$ is denoted by $h$. We have ignored the cross term for the heat kernel $\bar{K}$ in Eq.(2.19) corresponding to that for the new Hamiltonian density $\mathcal{H}^{\prime}$ in Eq.(2.13) under the near horizon approximation.

After performing the Gaussian integration with respect to $\Phi$, the free energy is obtained using the heat kernel representation [29] as

$$
\beta F=-\ln Z=-\frac{1}{2} \operatorname{Tr} \int_{0}^{\infty} \frac{d s}{s} \exp (-s \bar{K}) .
$$

The trace of the heat kernel is consist of two parts: the Euclidean time part and the space part. The Euclidean time part is evaluated by using the one dimensional eigenfunction of $-i \partial_{\tau}$ as

$$
\begin{aligned}
\operatorname{Tr} \exp \left(s \partial_{\tau}^{2}\right) & =\int_{0}^{\beta} d \tau \sum_{\ell=-\infty}^{\infty} \frac{1}{\beta} \exp \left(-s\left(\frac{2 \pi \ell}{\beta}\right)^{2}\right) \\
& =\sum_{n=-\infty}^{\infty} \frac{\beta}{(4 \pi s)^{1 / 2}} \exp \left(-\frac{\beta^{2} n^{2}}{4 s}\right) .
\end{aligned}
$$

The Poisson's summation formula is used in the second equality. The space part of the trace is calculated by the asymptotic expansion method

$$
\begin{aligned}
& \operatorname{Tr} \exp \left(\frac{s}{g^{\tau \tau}}\left(\sum_{i, j=1}^{D-1} \frac{1}{\sqrt{h}} \partial_{i}\left(\sqrt{h} h^{i j} \partial_{j}\right)-m^{2}\right)\right) \\
= & \frac{1}{(4 \pi s)^{(D-1) / 2}} \sum_{k=0}^{\infty} \bar{B}_{k}(-s)^{k} \exp \left(-\frac{s m^{2}}{g^{\tau \tau}}\right),
\end{aligned}
$$

where $\bar{B}_{k}$ 's are the coefficient functions of the asymptotic expansion. The explicit lower contributions are

$$
\begin{aligned}
& \bar{B}_{0}=\int d^{D-1} x\left(g^{\tau \tau}\right)^{(D-1) / 2} h^{1 / 2} \\
& \bar{B}_{1}=\left(\frac{1}{4} \frac{D-2}{D-1}-\frac{1}{6}\right) \int d^{D-1} x\left(g^{\tau \tau}\right)^{(D-1) / 2} h^{1 / 2} \bar{R}
\end{aligned}
$$


where bar notation denotes the quantities in the optical space. ${ }^{3}$

The free energy is expressed by multiplying the two trace parts in the lowest order as

$$
\begin{aligned}
F & =-\int_{0}^{\infty} \frac{d s}{s} \frac{1}{(4 \pi s)^{D / 2}} \sum_{n=1}^{\infty} \exp \left(-\frac{\beta^{2} n^{2}}{4 s}\right) \bar{B}_{0} \exp \left(-\frac{m^{2} s}{g^{\tau \tau}}\right) \\
& =-\frac{1}{\beta^{D} \pi^{D / 2}} \int_{0}^{\infty} \frac{d t}{t} t^{D / 2} \mathrm{e}^{-t} \sum_{n=1}^{\infty} \frac{1}{n^{D}} \bar{B}_{0} \exp \left(-\frac{m^{2} \beta^{2} n^{2}}{4 t g^{\tau \tau}}\right)
\end{aligned}
$$

where the integration variable has been changed in second equality as $t=\beta^{2} n^{2} /(4 s)$. The vacuum energy term $(n=0)$ is subtracted in the sum in Eq.(2.25). The contribution of the scalar field mass term shown to be very small near the horizon. After neglecting the mass term, a compact expression for the free energy is obtained

$$
F=-\frac{\zeta(D) \Gamma(D / 2)}{\pi^{D / 2} \beta^{D}} \bar{V}
$$

where $\bar{V}$ denotes the volume of the optical space defined by

$$
\bar{V}=\bar{B}_{0} \times 1=\int d^{D-1} x\left(g^{\tau \tau}\right)^{(D-1) / 2} h^{1 / 2} .
$$

The entropy and the internal energy are obtained by

$$
\begin{aligned}
S & =-\beta^{2} \frac{\partial F}{\partial \beta}=\frac{\zeta(D) D \Gamma(D / 2)}{\pi^{D / 2} \beta^{D-1}} \bar{V}, \\
U & =F+\beta^{-1} S=\frac{\zeta(D)(D-1) \Gamma(D / 2)}{\pi^{D / 2} \beta^{D}} \bar{V} .
\end{aligned}
$$

The obtained thermal quantities: free energy, entropy and internal energy in multi-parameter rotating case are apparently same form with that obtained in non-rotating [18] or single-parameter rotating cases [28]. The multi-parameter effects are included in the inverse temperature $\beta$ and the optical volume $\bar{V}$, which will be evaluated in the next subsection.

The obtained thermal quantities in four-dimensional flat spacetime are coincide with the Stefan-Boltzmann's law and therefore are considered as the generalization of them to $D$-dimensional multi-parameter rotating spacetime.

\footnotetext{
${ }^{3}$ It is worthwhile to note that the lowest order contribution $\bar{B}_{0}$ in Eq. $(2.24)$ can also be derived by using the semi-classical momentum eigenfunction.
} 


\subsection{Generalized area law}

In this subsection, we evaluate the generalized entropy formula (2.28) to the case of the multi-parameter rotation black holes. We first define the horizon and introduce Rindler space to evaluate the temperature and to define the invariant regularization parameter. The dominant contribution to the entropy comes from the near horizon region of the optical volume, which lead to the area law in general form.

$\diamond$ Metric Condition 3:

The horizon $r_{\mathrm{H}}$ is defined as the position of simple zeros for the inverse Euclidean metric components $1 / g^{\tau \tau}$ and $1 / g_{r r}$ :

$$
\left.\frac{1}{g^{\tau \tau}} \simeq \partial_{r} \frac{1}{g^{\tau \tau}(\theta)}\right|_{r_{H}} \times\left(r-r_{H}\right) \quad,\left.\quad \frac{1}{g_{r r}} \simeq \partial_{r} \frac{1}{g_{r r}(\theta)}\right|_{r_{H}} \times\left(r-r_{H}\right) .
$$

For outer horizon these coefficients are required to be positive, which exclude the cosmological horizon.

Next we set up to introduce Rindler space. We rewrite the Euclidean line element in order not to appear the cross term between time and azimuthal angle parts explicitly as

$$
\begin{aligned}
d s^{2}= & g_{\tau \tau} d \tau^{2}+2 \sum_{a=1}^{p} g_{\tau \varphi_{a}} d \tau d \varphi_{a}+\sum_{m, n=1}^{q} g_{\theta_{m} \theta_{n}} d \theta_{m} d \theta_{n}+g_{r r} d r^{2} \\
= & \frac{1}{g^{\tau \tau}} d \tau^{2}+\sum_{a, b=1}^{p} g_{\varphi_{a} \varphi_{b}}\left(d \varphi_{a}-\Omega_{a} d \tau\right)\left(d \varphi_{b}-\Omega_{b} d \tau\right) \\
& +\sum_{m, n=1}^{q} g_{\theta_{m} \theta_{n}} d \theta_{m} d \theta_{n}+g_{r r} d r^{2}
\end{aligned}
$$

The derivation of Eq.(2.31) is given in appendix C. The line element is now in the block diagonal form with respect to new azimuthal angles $d \varphi_{a}-\Omega_{a} d \tau$. We introduce a new radial variable $R$, which is invariant under the radial coordinate transformation, defined by

$$
R(r):=\int_{r_{\mathrm{H}}}^{r} d r \sqrt{g_{r r}}
$$


Using the new radial coordinate $R$, the line element (2.31) is expressed near the horizon in the form of Rindler spacetime as

$$
\left.d s^{2} \simeq \frac{1}{4} \partial_{r} \frac{1}{g^{\tau \tau}(\theta)} \partial_{r} \frac{1}{g_{r r}(\theta)}\right|_{r_{H}} R^{2} d \tau^{2}+d R^{2}+(\varphi, \theta \text { terms }) .
$$

$\diamond$ Metric Condition 4:

The temperature on the horizon $\left(T_{H}=1 / \beta_{H}\right)$ is defined by the conditions that no conical singularity and no angle dependence are required in Rindler space:

$$
\frac{2 \pi}{\beta_{H}}=\left(\left.\frac{1}{4} \partial_{r} \frac{1}{g^{\tau \tau}(\theta)} \partial_{r} \frac{1}{g_{r r}(\theta)}\right|_{r_{H}}\right)^{1 / 2}=\text { independent on } \theta \text {. }
$$

Under the definitions of the horizon (2.30) and the temperature (2.34), we consider the volume of the optical space $\bar{V}$ in Eq.(2.27). As $\bar{V}$ is divergent on the horizon, we introduce the invariant regularization parameter for the small distance $\epsilon_{\mathrm{inv}}$ and the large distance $L_{\text {inv }}$ in the Rindler spacetime as

$$
\epsilon_{\mathrm{inv}}:=\int_{r_{H}}^{r_{H}+\epsilon(\theta)} d r\left(g_{r r}(\theta)\right)^{1 / 2} \quad, \quad L_{\mathrm{inv}}:=\int_{r_{H}}^{r_{\mathrm{H}}+L(\theta)} d r\left(g_{r r}(\theta)\right)^{1 / 2}
$$

The regularization parameters in the Rindler spacetime $\epsilon_{\mathrm{inv}}, L_{\mathrm{inv}}$ are required angle independent, while those in the original spacetime $\epsilon(\theta), L(\theta)$ become angle dependent. This is because the physical quantities in Rindler space should be angle independent just as the temperature, for they are invariant under the radial coordinate transformation and then observer independent. ${ }^{4}$ Using these regularization parameters, the radial integration for the optical volume $\bar{V}$ is evaluated via Rindler space near the horizon as

$$
\begin{aligned}
& \left.\int_{r_{H}+\epsilon(\theta)}^{r_{\mathrm{H}}+L(\theta)} d r\left(g^{\tau \tau}\right)^{D / 2-1 / 2}\left(g_{r r}\right)^{1 / 2}\right|_{r_{\mathrm{H}}} \\
= & \left.\int_{\epsilon_{\mathrm{inv}}}^{L_{\mathrm{inv}}} d R\left(g^{\tau \tau}\right)^{D / 2-1 / 2}\right|_{r_{\mathrm{H}}} \simeq\left(\frac{\beta_{H}}{2 \pi}\right)^{D / 2-1 / 2} \frac{1}{(D-2) \epsilon_{\mathrm{inv}}^{D-2}},
\end{aligned}
$$

\footnotetext{
${ }^{4}$ Note that the regularization parameter is angle independent for non-rotating case in the brick wall model [16].
} 
where the temperature condition (2.34) is used. The contribution from the large regularization parameter $\left(L_{\text {inv }}\right)$ is negligible under the magnitude condition: $\epsilon_{\mathrm{inv}} \ll L_{\mathrm{inv}}$. The angular integration in $\bar{V}$ gives the area on the horizon as

$$
A_{H}:=\left.\int \prod_{a=1}^{p} d \varphi_{a} \prod_{m=1}^{q} d \theta_{m} \sqrt{\operatorname{det}\left(g_{\varphi_{a} \varphi_{b}}\right) \operatorname{det}\left(g_{\theta_{m} \theta_{n}}\right)}\right|_{r_{H}} .
$$

Combining together the radial coordinate integration (2.36) and the angular integration (2.37), the optical volume $\bar{V}(2.27)$ is evaluated as

$$
\bar{V}=\left(\frac{\beta_{\mathrm{H}}}{2 \pi}\right)^{D-1} \frac{A_{H}}{(D-2) \epsilon_{\mathrm{inv}}^{D-2}} .
$$

Then the generalized entropy formula (2.28) is evaluated near the horizon using Eq. (2.38) as

$$
S=\frac{\zeta(D) D \Gamma(D / 2-1)}{2^{D} \pi^{3 D / 2-1}} \frac{A_{H}}{\epsilon_{\mathrm{inv}}^{D-2}}
$$

We have obtained the generalized area law for quantum scalar field under the multi-parameter rotating black hole spacetime without using the explicit form of black hole solutions. The expression of the area law under multi-parameter cases has the same form as that under non-rotating and single-parameter rotating cases [28]. Therefore we can take smooth non-rotating limit of the generalized are law. The rotating effects are included in the temperature and the volume of the optical space.

The thermal quantities; entropy $S$, internal energy $U$ and Helmholtz's free energy $F$ are consist of the product form of the temperature $(\beta)$ term and the volume term $(\bar{V})$ in the optical space. The contribution of the real space integration in $\bar{V}$ is extremely large near the horizon region and then the area law of the black hole entropy is derived.

We also note that the super-radiant modes are take into account in our entropy expression (2.39), because the scalar field energy $E$ can be negative if the angular momentum $P_{\varphi_{a}}$ be negative and satisfies our energy restriction (2.15): $H-\sum_{a=1}^{p} \Omega_{a} P_{\varphi_{a}} \geq 0$. 


\section{Application}

In this section, we apply the generalized area law under the multi-parameter rotating black hole spacetime to the cases of Kerr-AdS black hole spacetime, which are interesting and developing recently. We first study the four-dimensional KerrAdS spacetime as single-parameter rotating case and next the five-dimensional Kerr-AdS spacetime as double-parameter rotating case. A special feature in the Kerr-(anti)-de Sitter spacetime is that the rotation effects remain even in the asymptotic region for rotating black holes. In general $D$ dimension, the multiparameter rotating black hole solutions are known to exist systematically for the even- and odd-dimensional cases respectively. The examples in this section may give the understanding for the general multi-parameter rotating black hole cases.

\subsection{Kerr-AdS black holes in four dimensions}

The four-dimensional Kerr-AdS metric is given by Carter [30] as

$$
\begin{aligned}
d s_{D=4}^{2}= & -\frac{\Delta}{\rho^{2}}\left(d t-\frac{a \sin ^{2} \theta}{\Xi} d \varphi\right)^{2}+\frac{\rho^{2}}{\Delta} d r^{2}+\frac{\rho^{2}}{\Delta_{\theta}} d \theta^{2} \\
& +\frac{\Delta_{\theta} \sin ^{2} \theta}{\rho^{2}}\left(a d t-\frac{r^{2}+a^{2}}{\Xi} d \varphi\right)^{2}
\end{aligned}
$$

with

$$
\begin{aligned}
\Delta & =\left(r^{2}+a^{2}\right)\left(1+r^{2} \ell^{2}\right)-2 M r, \quad \Delta_{\theta}=1-a^{2} \ell^{2} \cos ^{2} \theta, \\
\rho^{2} & =r^{2}+a^{2} \cos ^{2} \theta, \quad \Xi=1-a^{2} \ell^{2},
\end{aligned}
$$

where $M, a$ and $\Lambda=-3 \ell^{-2}$ denote the black hole mass, its angular momentum per unit mass and the cosmological term respectively. In order to apply the general area law (2.39), the metric (3.1) is rewritten in the general form:

$$
d s_{D=4}^{2}=g_{t t} d t^{2}+2 g_{t \varphi} d t d \varphi+g_{\varphi \varphi} d \varphi^{2}+g_{\theta \theta} d \theta^{2}+g_{r r} d r^{2}
$$

where each components are given by

$$
\begin{aligned}
g_{t t} & =\frac{1}{\rho^{2}}\left(-\Delta+\Delta_{\theta} a^{2} \sin ^{2} \theta\right), \quad g_{t \varphi}=\frac{a \sin ^{2} \theta}{\rho^{2} \Xi}\left(\Delta-\left(r^{2}+a^{2}\right) \Delta_{\theta}\right), \\
g_{\varphi \varphi} & =\frac{\sin ^{2} \theta}{\rho^{2} \Xi^{2}}\left(-\Delta a^{2} \sin ^{2} \theta+\left(r^{2}+a^{2}\right)^{2} \Delta_{\theta}\right), g_{\theta \theta}=\frac{\rho^{2}}{\Delta}, g_{r r}=\frac{\rho^{2}}{\Delta_{\theta}} .
\end{aligned}
$$


In order to obtain the temperature and the angular velocity we need to know some of the inverse components of the metric:

$$
g^{t t}=\frac{g_{\varphi \varphi}}{\Gamma}, \quad g^{\varphi \varphi}=\frac{g_{t t}}{\Gamma}, \quad g^{t \varphi}=-\frac{g_{t \varphi}}{\Gamma},
$$

with $\Gamma:=g_{t t} g_{\varphi \varphi}-g_{t \varphi}^{2}=-\Delta \Delta_{\theta} \sin ^{2} \theta / \Xi^{2}$. The horizon $r_{H}$ is defined as the larger zero of $1 / g^{t t}$ as well as $1 / g_{r r}$, which gives $\Delta\left(r=r_{H}\right)=0$. The angular velocity and the temperature on the horizon are calculated as

$$
\begin{aligned}
& \Omega_{H}=\left.\frac{g^{t \varphi}}{g^{\varphi \varphi}}\right|_{r_{H}}=-\left.\frac{g_{t \varphi}}{g_{\varphi \varphi}}\right|_{r_{H}}=\frac{a \Xi}{r_{H}^{2}+a^{2}}, \\
& \frac{2 \pi}{\beta_{H}}=\left.\frac{\partial_{r} \Delta}{2\left(r^{2}+a^{2}\right)}\right|_{r_{H}}=\frac{r_{H}}{2\left(r_{H}^{2}+a^{2}\right)}\left(1+3 r_{H}^{2} \ell^{-2}+a^{2} \ell^{-2}-\frac{a^{2}}{r_{H}^{2}}\right) .
\end{aligned}
$$

The angular momentum $\Omega_{H}$ is the same form as that given by Hawking, Hunter and Taylor-Robinson. Other definition of angular momentum given by Gibbons, Perry and Pope will be discussed in the next section. The entropy for the quantum scalar field is given by

$$
S_{D=4}=\frac{1}{360 \pi} \frac{A_{H}}{\epsilon_{\mathrm{inv}}^{2}}
$$

with the area on the horizon $A_{H}=4 \pi\left(r_{H}^{2}+a^{2}\right) / \Xi$. The resultant area law (3.8) recovers that of the previous work for Kerr black hole case [28].

\subsection{Kerr-AdS black holes in five dimensions}

In five-dimensional Einstein's field equation, the double-parameter rotating black hole solution holds, which is an example of multi-parameter black hole spacetime. The double-parameter solution is more complicated than single-parameter solution with the existence of the cross-metric term between two azimuthal angles.

The metric of the Kerr-AdS black hole in five dimensions is given by Hawking, Hunter and Taylor-Robinson [5] as

$$
\begin{aligned}
d s_{D=5}^{2}= & -\frac{\Delta}{\rho^{2}}\left(d t-\frac{a \sin ^{2} \theta}{\Xi_{a}} d \varphi_{a}-\frac{b \cos ^{2} \theta}{\Xi_{b}} d \varphi_{b}\right)^{2} \\
& +\frac{\Delta_{\theta} \sin ^{2} \theta}{\rho^{2}}\left(a d t-\frac{\left(r^{2}+a^{2}\right)}{\Xi_{a}} d \varphi_{a}\right)^{2}
\end{aligned}
$$




$$
\begin{aligned}
& +\frac{\Delta_{\theta} \cos ^{2} \theta}{\rho^{2}}\left(b d t-\frac{\left(r^{2}+b^{2}\right)}{\Xi_{b}} d \varphi_{b}\right)^{2} \\
& +\frac{\rho^{2}}{\Delta} d r^{2}+\frac{\rho^{2}}{\Delta_{\theta}} d \theta^{2}+\frac{\left(1+r^{2} \ell^{2}\right)}{r^{2} \rho^{2}}(a b d t \\
& \left.-\frac{b\left(r^{2}+a^{2}\right) \sin ^{2} \theta}{\Xi_{a}} d \varphi_{a}-\frac{a\left(r^{2}+b^{2}\right) \cos ^{2} \theta}{\Xi_{b}} d \varphi_{b}\right),
\end{aligned}
$$

where

$$
\begin{aligned}
\Delta & =\frac{1}{r^{2}}\left(r^{2}+a^{2}\right)\left(r^{2}+b^{2}\right)\left(1+r^{2} \ell^{2}\right)-2 M, \\
\Delta_{\theta} & =\left(1-a^{2} \ell^{2} \cos ^{2} \theta-b^{2} \ell^{2} \sin ^{2} \theta\right), \\
\rho^{2} & =\left(r^{2}+a^{2} \cos ^{2} \theta+b^{2} \sin ^{2} \theta\right) \\
\Xi_{a} & =\left(1-a^{2} \ell^{2}\right), \quad \Xi_{b}=\left(1-b^{2} \ell^{2}\right) .
\end{aligned}
$$

The notations $M, a, b$ and $\Lambda=-6 \ell^{-2}$ denote the mass of black hole, two angular velocities and negative cosmological term. In order to apply the generalized area law (2.39), we rewrite the metric in the general form:

$$
\begin{aligned}
d s_{D=5}^{2}= & g_{t t} d t^{2}+2\left(g_{t \varphi_{a}} d t d \varphi_{a}+g_{t \varphi_{a}} d t d \varphi_{a}\right) \\
& +g_{\varphi_{a} \varphi_{a}} d \varphi_{a}^{2}+g_{\varphi_{b} \varphi_{b}} d \varphi_{b}^{2}+2 g_{\varphi_{a} \varphi_{b}} d \varphi_{a} d \varphi_{b} \\
& +g_{\theta \theta} d \theta^{2}+g_{r r} d r^{2}
\end{aligned}
$$

where each components are

$$
\begin{aligned}
g_{t t} & =\frac{1}{\rho^{2}}\left(-\Delta+\Delta_{\theta}\left(a^{2} \sin ^{2} \theta+b^{2} \cos ^{2} \theta\right)+\Delta_{\ell} a^{2} b^{2}\right), \\
g_{t \varphi_{a}} & =\frac{a \sin ^{2} \theta}{\rho^{2} \Xi_{a}}\left(\Delta-A\left(\Delta_{\theta}+\Delta_{\ell} b^{2}\right)\right), \\
g_{t \varphi_{b}} & =\frac{b \cos ^{2} \theta}{\rho^{2} \Xi_{b}}\left(\Delta-B\left(\Delta_{\theta}+\Delta_{\ell} a^{2}\right)\right), \\
g_{\varphi_{a} \varphi_{a}} & =\frac{\sin ^{2} \theta}{\rho^{2} \Xi_{a}}\left(-\Delta a^{2} \sin ^{2} \theta+A^{2}\left(\Delta_{\theta}+\Delta_{\ell} b^{2} \sin ^{2} \theta\right)\right), \\
g_{\varphi_{b} \varphi_{b}} & =\frac{\cos ^{2} \theta}{\rho^{2} \Xi_{b}}\left(-\Delta b^{2} \cos ^{2} \theta+B^{2}\left(\Delta_{\theta}+\Delta_{\ell} a^{2} \cos ^{2} \theta\right)\right), \\
g_{\varphi_{a} \varphi_{b}} & =\frac{a b \sin ^{2} \theta \cos ^{2} \theta}{\rho^{2} \Xi_{a} \Xi_{b}}\left(-\Delta+A B \Delta_{\ell}\right),
\end{aligned}
$$


where some additional notations are defined as

$$
A=r^{2}+a^{2}, \quad B=r^{2}+b^{2}, \quad \Delta_{\ell}=r^{-2}+\ell^{-2} .
$$

We need the contravariant components of the metric with respect to time and azimuthal angle to obtain the angular velocity and temperature:

$$
\begin{aligned}
g^{t t}= & \frac{1}{r^{2} \rho^{2} \Delta_{\theta}}\left(A b^{2} \cos ^{2} \theta+B a^{2} \sin ^{2} \theta-\ell^{-2} a^{2} b^{2}\left(A \cos ^{2} \theta+B \sin ^{2} \theta\right)\right) \\
& -\frac{1}{r^{4} \rho^{2} \Delta} A^{2} B^{2} \\
g^{t \varphi_{a}}= & \frac{a \Xi_{a}}{r^{2} \rho^{2}}\left(\frac{1}{\Delta_{\theta}}\left(B-\ell^{-2} b^{2}\left(A \cos ^{2} \theta+B \sin ^{2} \theta\right)-\frac{1}{r^{2} \Delta} A B^{2}\right)\right. \\
g^{t \varphi_{b}}= & \frac{b \Xi_{b}}{r^{2} \rho^{2}}\left(\frac{1}{\Delta_{\theta}}\left(A-\ell^{-2} a^{2}\left(A \cos ^{2} \theta+B \sin ^{2} \theta\right)-\frac{1}{r^{2} \Delta} A^{2} B\right) .\right.
\end{aligned}
$$

The detailed derivation is given in appendix $\mathrm{D}$.

The horizon $r_{H}$ is defined as a larger zero of the inverse metric components $1 / g^{t t}$ and $1 / g_{r r}$, which is given by the outer real root of $\Delta$ :

$$
\Delta\left(r=r_{H}\right)=0 \Longleftrightarrow\left(r_{H}^{2}+a^{2}\right)\left(r_{H}^{2}+b^{2}\right)\left(1+r_{H}^{2} \ell^{-2}\right)=2 M r_{H}^{2},
$$

The two angular velocities on the horizon Eq.(2.11) are obtained:

$$
\Omega_{H_{a}}=\frac{g^{t \varphi_{a}}}{g^{t t}}=\frac{a \Xi_{a}}{r_{H}^{2}+a^{2}} \quad, \quad \Omega_{H_{b}}=\frac{g^{t \varphi_{b}}}{g^{t t}}=\frac{b \Xi_{b}}{r_{H}^{2}+b^{2}},
$$

which coincide with those by Hawking, Hunter and Taylor-Robinson [5]. It is worthwhile to note that the angular velocities $\Omega_{\mathrm{H}_{a}}=g^{t \varphi_{a}} / g^{t t}$ and $/$ or $\Omega_{\mathrm{H}_{b}}=$ $g^{t \varphi_{b}} / g^{t t}$ do not equal to $-g_{t \varphi_{a}} / g_{\varphi a \varphi a}$ and/or $-g_{t \varphi_{b}} / g_{\varphi b \varphi b}$ generally in the multiparameter case but they equal in single-parameter case due to the existence of $g_{\varphi_{a} \varphi_{b}}$ components. The inverse Hawking temperature on the horizon is given in Eq. (2.34) as

$$
\frac{2 \pi}{\beta_{H}}=\left.\frac{r^{2} \partial_{r} \Delta}{2 A B}\right|_{r_{H}}=r_{H}\left(1+r_{H}^{2} \ell^{-2}\right)\left(\frac{1}{r_{H}^{2}+a^{2}}+\frac{1}{r_{H}^{2}+b^{2}}\right)-\frac{1}{r_{H}} .
$$

The entropy of the five-dimensional Kerr-AdS black hole is obtained from the general expression in Eq.(2.36) as

$$
S_{D=5}=\frac{5 \zeta(5)}{2^{6} \pi^{6}} \frac{A_{H}}{\epsilon_{\mathrm{inv}}^{3}},
$$


where the area of the five-dimensional Kerr-AdS black hole is given by

$$
A_{H}=\frac{2 \pi^{2}\left(r_{H}^{2}+a^{2}\right)\left(r_{H}^{2}+b^{2}\right)}{r_{H} \Xi_{a} \Xi_{b}} .
$$

We have obtained quantum scalar field contribution to the entropy in the fourand five-dimensional spacetime, which will help to understand the cases in more higher-dimensional rotating black hole spacetime. For more higher dimensional black hole solutions, the additional number of rotating parameter can increase as the increase of each even dimension.

\section{Relation Between Thermodynamics for Scalar Field and Thermodynamics for Black Holes}

In this section we study the thermodynamics of scalar field which obtained in section 2 according to the standard statistical method under general form of metrics and compare them with the corresponding black hole thermodynamics.

First we can show that the statistical (Gibbs-Duhem) relation for the quantum scalar field holds as

$$
T S=U+p \bar{V}
$$

where the volume of the optical space $\bar{V}$ is given in Eq.(2.27), the entropy $S$ in Eq.(2.28), the internal energy $U$ in Eq.(2.29) and the pressure $p$ is given by

$$
p:=-\frac{\partial F}{\partial \bar{V}}=\frac{\zeta(D) \Gamma(D / 2)}{\pi^{D / 2} \beta_{H}^{D}} .
$$

The first law of thermodynamics also holds as

$$
T d S=d U+p d \bar{V}
$$

The statistical relation (4.1) and the first law of thermodynamics (4.2) are related through scaling property. These thermodynamic relations hold because we have derived the thermal quantities according to the standard statistical mechanics method under general form of the black hole metrics. 
In order to compare the thermodynamics for the scalar field to those for black holes, we define the effective gravitational constant of the scalar field

$$
G^{\prime}=\frac{\pi^{3 D / 2-1} 2^{D} \epsilon^{D-2}}{4 \zeta(D) D \Gamma(D / 2-1)},
$$

which normalizes the entropy as $S=A_{H} / 4 G^{\prime}$.

We can express the thermal quantities in the language of black hole variables from that of scalar field variables if the explicit black hole solutions is used for the general form of the background metric. In four-dimensional Karr-AdS black hole case, which was studied in subsection 3.1, the statistical relation in the language of black holes is obtained in the form:

$$
T S=\frac{E_{H}}{2}-\Omega_{H} J+X_{H} \ell^{-2},
$$

where the black hole energy $E_{H}$, angular momentum $J$ and the conjugate variable to cosmological term $X_{H}$ are defined:

$$
E_{H}=\frac{M}{\Xi G^{\prime}}, \quad J=\frac{M a}{\Xi^{2} G^{\prime}}, \quad X_{H}=\frac{r_{H}\left(r_{H}^{2}+a^{2}\right)}{2 \Xi G^{\prime}} .
$$

Temperature $T$ and entropy $S$ in Eq.(4.5) are the same as in Eq.(4.1). The first law of the thermodynamics can also be expressed in language of black holes as

$$
T d S=d E_{G}-\Omega_{G} d J-X_{G} d \ell^{-2},
$$

where the black hole energy $E_{G}$, angular velocity $\Omega_{G}$ and conjugate variable to cosmological term $X_{G}$ are defined:

$$
\begin{aligned}
E_{G} & =\frac{M}{\Xi^{2} G^{\prime}}, \quad \Omega_{G}=\frac{a\left(1+r_{H}^{2} \ell^{-2}\right)}{r_{H}^{2}+a^{2}}, \\
X_{G} & =\frac{r_{H}\left(r_{H}^{2}+a^{2}\right)}{2 \Xi^{2} G^{\prime}}\left(1+\frac{a^{2}}{2 r_{H}^{2}}\left(1-r_{H}^{2} \ell^{-2}\right)\right) .
\end{aligned}
$$

The coefficients in front of each thermal quantities are from the scaling property; 1/2 for energy, 1 for thermal and angular terms and -1 for conjugate to cosmological term. The differences between $E_{H}, \Omega_{H}, X_{H}$ in Eq.(4.6) and $E_{G}, \Omega_{G}, X_{G}$ in Eq.(4.8) are due to the non-linearity in scaling transformation in each quantities. 
We should note the difference between the angular velocity defined by Hawking, Hunter and Taylor-Robbinson $\Omega_{H}$ in Eq.(3.6) [5] and that by Gibbons, Perry and Pope $\Omega_{G}$ in Eq.(4.8) [6]. The angular velocity $\Omega_{H}$ is considered to be measured relative to a rotating frame at infinity, while the angular velocity $\Omega_{G}$ is measured relative to a non-rotating frame at infinity:

$$
\Omega_{G}:=\Omega_{H}-\Omega_{\infty}=\frac{a\left(1+r_{H}^{2} \ell^{-2}\right)}{r_{H}^{2}+a^{2}},
$$

with $\Omega_{\infty}=-a \ell^{-2}$. Gibbons, Perry and Pope [6] stressed the importance to hold the first law of thermodynamics, in which $\Omega_{G}$ as well as $E_{G}$ are used in Eq.(3.8).

The naive derivation of the conjugate variable to cosmological term is the cosmological term in the action integration:

$$
-\frac{1}{16 \pi G} \int d^{4} x \sqrt{-g} 2 \Lambda=\beta_{H} \frac{r_{H}\left(r_{H}^{2}+a^{2}\right)}{2 \Xi G} \ell^{-2}
$$

where the gravitational constant is recovered in Eq.(4.10), which is replaced by $G^{\prime}$ in $X_{H}$ in Eq.(4.6). For the case of the constant cosmological term; $d \ell^{-2}=0$, the first law (4.7) reduces to that given by Gibbons, Perry and Pope with the replacement: $G \rightarrow G^{\prime}$. We include the cosmological term in order to keep the scaling transformation.

By this correspondence, the thermodynamics for the quantum scalar fields around black holes is consistent with that for the black hole itself.

\section{$5 \quad$ Summary and Discussions}

We have studied the statistical mechanics for quantum scalar fields under multiparameter rotating black hole spacetime in arbitrary $D$ dimensions. We have obtained the generalized area law for scalar field entropy around the black holes under the general form of the metric. The conditions imposed on the metric are the following.

$\diamond 1$ Off-diagonal components between time and azimuthal angles $g_{t \varphi_{a}}$ and among azimuthal angles $g_{\varphi_{a} \varphi_{b}}$ are assumed to exist. This condition indicates multiparameter rotations with velocities: $\Omega_{H_{a}}=g^{t \varphi_{a}} / g^{t t}$.

$\diamond 2$ Any metric components are assumed not to be functions of $t$ and $\varphi_{a}$. This condition ensures the energy and angular momentum conservations. 
$\diamond 3$ The inverse of metric components; $1 / g^{t t}$ and $1 / g_{r r}$ are assumed to have simple zeros at horizon position $r_{H}$.

$\diamond 4$ The product of the inverse metric components; $\partial_{r}\left(1 / g^{t t}\right) \times \partial_{r}\left(1 / g_{r r}\right)$ is assumed not to depend on angle $\theta$ on the horizon $r_{H}$. This condition leads the well-defined temperature on $r_{H}$.

The contribution to the scalar field entropy is extremely dominant near the horizon in the optical volume. We introduce regularization parameter $\epsilon_{\text {inv }}$ in the Rindler spacetime in order to control the divergence on the horizon. The large contribution near the horizon leads the area law of the entropy for the scalar fields naturally.

The thermodynamics for the scalar fields can be expressed in the language of the black hole variables, where the gravitational constant is replaced by effective one of the scalar field; $G \rightarrow G^{\prime}$.

In deriving the area law for the quantum scalar field under the multi-parameter rotating black hole spacetime, we have postulated the quasi-equilibrium state for quantum scalar fields. In connection with this, we should comment on the superradiant instability for Kerr black hole spacetime in $(3+1)$ dimensions [31, 32, 33]. Super-radiant effects occur also in our analysis through the energy restriction, because scalar field energy can be negative if the scalar field angular momentum is in the inverse direction with respect to the direction of the black hole angular momentum. The relation between our quasi-equilibrium treatment and the superradiant instability phenomena will be studied in our future work.

The quantum effects of gravity near the horizon is very important in deriving the black hole entropy and they will be our future problem too.

\section{Acknouledgements}

The authors wish to thank Dr. K. Shigemoto for his careful reading of the manuscript and useful comments.

\section{Appendix}




\section{A Derivation of Null Potency of $\eta$ in Eq.(2.10)}

In this appendix, we calculate the square of newly introduced Killing vector $\eta\left(=\xi_{t}+\sum_{a=1}^{p} \Omega_{H_{a}} \xi_{\varphi_{a}}\right)$ and show its null potency. First we note the orthogonality relation among metrics:

$$
g_{t t} g^{t t}+\sum_{a=1}^{p} g_{t \varphi_{a}} g^{t \varphi_{a}}=1, g_{t \varphi_{a}} t^{t t}+\sum_{b=1}^{p} g_{\varphi_{a} \varphi_{b}} g^{t \varphi_{b}}=0 .
$$

Using orthogonality relations, we obtain the metric-angular velocity relations:

$$
\begin{aligned}
& g_{t t}+2 \sum_{a=1}^{p} g_{t \varphi_{a}} \Omega_{a}+\sum_{a, b=1}^{p} g_{\varphi_{a} \varphi_{b}} \Omega_{a} \Omega_{b}=\frac{1}{g^{t t}}, \\
& -2 \sum_{a=1}^{p} g_{t \varphi_{a}} \Omega_{a}-\sum_{a, b=1}^{p} g_{\varphi_{a} \varphi_{b}} \Omega_{a} \Omega_{b}=\sum_{a, b=1}^{p} g_{\varphi_{a} \varphi_{b}} \Omega_{a} \Omega_{b}, \\
& \sum_{a=1}^{p} g_{t \varphi_{a}} \Omega_{\mathrm{H} a}=-\sum_{a, b=1}^{p} g_{\varphi_{a} \varphi_{b}} \Omega_{\mathrm{H} a} \Omega_{b}
\end{aligned}
$$

Then we evaluate square of $\eta$, in the following, as

$$
\begin{aligned}
\eta^{2} & =g_{t t}+2 \sum_{a=1}^{p} g_{t \varphi_{a}} \Omega_{\mathrm{H} a}+\sum_{a, b=1}^{p} g_{\varphi_{a} \varphi_{b}} \Omega_{\mathrm{H} a} \Omega_{\mathrm{H} b} \\
& =g_{t t}+2 \sum_{a=1}^{p} g_{t \varphi_{a}} \Omega_{\mathrm{H} a}+\sum_{a, b=1}^{p} g_{\varphi_{a} \varphi_{b}} \Omega_{\mathrm{H} a} \Omega_{\mathrm{H} b} \\
& +2 \sum_{a=1}^{p} g_{t \varphi_{a}} \Omega_{a}+\sum_{a, b=1}^{p} g_{\varphi_{a} \varphi_{b}} \Omega_{a} \Omega_{b}-2 \sum_{a=1}^{p} g_{t \varphi_{a}} \Omega_{a}-\sum_{a, b=1}^{p} g_{\varphi_{a} \varphi_{b}} \Omega_{a} \Omega_{b} \\
& =\frac{1}{g^{t t}}+\sum_{a, b=1}^{p} g_{\varphi_{a} \varphi_{b}} \Omega_{a} \Omega_{b}-2 \sum_{a, b=1}^{p} g_{\varphi_{a} \varphi_{b}} \Omega_{\mathrm{H} a} \Omega_{b}+2 \sum_{a, b=1}^{p} g_{\varphi_{a} \varphi_{b}} \Omega_{\mathrm{H} a} \Omega_{\mathrm{H} b} \\
& =\frac{1}{g^{t t}}+\sum_{a, b=1}^{p} g_{\varphi_{a} \varphi_{b}}\left(\Omega_{\mathrm{H} a}-\Omega_{a}\right)\left(\Omega_{\mathrm{H} b}-\Omega_{b}\right)
\end{aligned}
$$

where we have used the metric-angular velocity relation of Eqs.(A.2)-(A.4) in the second equality to show the third equality. The expression of $\eta^{2}$ in Eq.(2.10) is the last equality in Eq.(A.5), which shows the null potency at horizon position $r=r_{H}$, where $\Omega_{\mathrm{H} a}=\Omega_{a}$ and $1 / g^{t t}=0$. 


\section{B Derivation of Positivity of $\mathcal{H}^{\prime}$ in Eq.(2.4)}

In order to show the positivity of newly introduced energy density $\mathcal{H}^{\prime}$ of Eq. (2.13), we insert the explicit expression of scalar fields in the Lagrangian density (2.4) and rewrite as

$$
\begin{aligned}
\mathcal{H}^{\prime}= & \Pi \partial_{t} \Phi-\mathcal{L}_{\text {scalar }}+\sum_{a=1}^{p} \Omega_{\mathrm{H} a} \Pi \partial_{\varphi_{a}} \Phi \\
= & \Pi \partial_{t} \Phi+\frac{1}{2}\left(g^{t t}\left(\partial_{t} \Phi\right)^{2}+2 \sum_{a=1}^{p} g^{t \varphi_{a}} \partial_{t} \Phi \partial_{\varphi_{b}} \Phi+\sum_{a, b=1}^{p} g^{\varphi_{a} \varphi_{b}} \partial_{\varphi_{a}} \Phi \partial_{\varphi_{b}} \Phi\right. \\
& +(r, \theta \text { and mass terms }))+\sum_{a=1}^{p} \Omega_{\mathrm{H} a} \Pi \partial_{\varphi_{a}} \Phi \\
= & \frac{1}{2}\left(-g^{t t}\left(\partial_{t} \Phi\right)^{2}+\sum_{a, b=1}^{p} g^{\varphi_{a} \varphi_{b}} \partial_{\varphi_{a}} \Phi \partial_{\varphi_{b}} \Phi+(r, \theta \text { and mass terms })\right) \\
& +\sum_{a=1}^{p} \Omega_{\mathrm{H} a} \Pi \partial_{\varphi_{a}} \Phi .
\end{aligned}
$$

After eliminate the time derivative terms by means of the momentum (2.5), we obtain

$$
\begin{aligned}
\mathcal{H}^{\prime}= & \frac{1}{2}\left(-\frac{1}{g^{t t}}\left(\Pi+\sum_{a=1}^{p} g^{t \varphi_{a}} \partial_{\varphi_{a}} \Phi\right)^{2}+\sum_{a, b=1}^{p} g^{\varphi_{a} \varphi_{b}} \partial_{\varphi_{a}} \Phi \partial_{\varphi_{b}} \Phi\right. \\
& +(r, \theta \text { and mass terms }))+\sum_{a=1}^{p} \Omega_{\mathrm{H} a} \Pi \partial_{\varphi_{a}} \Phi \\
= & \frac{1}{2}\left(-\frac{1}{g^{t t}} \Pi^{2}+\sum_{a, b=1}^{p} \tilde{g}^{\varphi_{a} \varphi_{b}} \partial_{\varphi_{a}} \Phi \partial_{\varphi_{b}} \Phi+(r, \theta \text { and mass terms })\right) \\
& +\sum_{a=1}^{p}\left(\Omega_{\mathrm{H} a}-\Omega_{a}\right) \Pi \partial_{\varphi_{a}} \Phi
\end{aligned}
$$

where $\tilde{g}^{\varphi_{a} \varphi_{b}}$ is the inverse metric of $g_{\varphi_{a} \varphi_{b}}$ defined as

$$
g_{\varphi_{a} \varphi_{b}} \tilde{g}^{\varphi_{b} \varphi_{c}}=\delta_{a}^{c} \quad, \quad \tilde{g}^{\varphi_{a} \varphi_{b}}:=g^{\varphi_{a} \varphi_{b}}-\frac{g^{t \varphi_{a}} g^{t \varphi_{b}}}{g^{t t}}
$$


More conveniently we introduce the inverse metric $h^{i j}$ of special part metrics $h_{i j}$ defined by

$$
h^{i j}:=\left(\tilde{g}^{\varphi_{a} \varphi_{b}}, g^{\theta_{m} \theta_{m}}=\frac{1}{g_{\theta_{m} \theta_{m}}}, g^{r r}=\frac{1}{g_{r r}}\right),
$$

which is the expression of the inverse metric (2.14). The desired form for $\mathcal{H}^{\prime}$ of Eq.(2.13) is obtained:

$$
\mathcal{H}^{\prime}=\frac{1}{2}\left(-\frac{\Pi^{2}}{g^{t t}}+\sum_{i, j}^{D-1} h^{i j} \partial_{i} \Phi \partial_{j} \Phi+\mu^{2} \Phi^{2}\right)+\sum_{a=1}^{p}\left(\Omega_{\mathrm{H} a}-\Omega_{a}\right) \Pi \partial_{\varphi_{a}} \Phi,
$$

where this new Hamiltonian density is positive definite under the near horizon approximation $\Omega_{\mathrm{H} a} \simeq \Omega_{a}$.

\section{Derivation of Line Element in Eq.(2.31)}

Using the definition of angular velocity (2.11) and orthogonality relation (A.1), we obtain another metric-angular momentum identities:

$$
\begin{aligned}
g_{t t} & =\frac{1}{g^{t t}}+\sum_{a, b=1}^{p} g_{\varphi_{a} \varphi_{b}} \Omega_{\varphi_{a}} \Omega_{\varphi_{b}}, \\
g_{t \varphi_{a}} & =-\sum_{b=1}^{p} g_{\varphi_{a} \varphi_{b}} \Omega_{\varphi_{b}} .
\end{aligned}
$$

Then the line element in the original form (2.2) is rewritten using the identities as

$$
\begin{aligned}
d s^{2}= & g_{\tau \tau} d \tau^{2}+2 \sum_{a=1}^{p} g_{\tau \varphi_{a}} d \tau d \varphi_{a}+(r, \theta \text { terms }), \\
= & \left(\frac{1}{g^{\tau \tau}}+\sum_{a, b=1}^{p} g_{\varphi_{a} \varphi_{b}} \Omega_{\varphi_{a}} \Omega_{\varphi_{b}}\right) d \tau^{2}-2 \sum_{a, b=1}^{p} g_{\varphi_{a} \varphi_{b}} \Omega_{b} d t d \varphi_{a} \\
& +\sum_{a, b=1}^{p} g_{\varphi_{a} \varphi_{b}} d \varphi_{a} d \varphi_{b}+(r, \theta \text { terms }) \\
= & \frac{1}{g^{\tau \tau}} d \tau^{2}+\sum_{a, b=1}^{p} g_{\varphi_{a} \varphi_{b}}\left(d \varphi_{a}-\Omega_{a} d \tau\right)\left(d \varphi_{b}-\Omega_{b} d \tau\right) \\
& +(r, \theta \text { terms })
\end{aligned}
$$

which complete the derivation of Eq.(2.31). 


\section{Contravariant Metric Components for Kerr- AdS Black Holes in Five Dimensions}

We derive the contravariant metric components with respect to time and azimuthal angle to calculate the area law of the black hole entropy, which are defined in general form as

$$
g^{t t}=\frac{\gamma_{t}}{\Gamma} \quad, \quad g^{t \varphi_{a}}=\frac{\gamma_{\varphi_{a}}}{\Gamma} \quad, \quad g^{t \varphi_{b}}=\frac{\gamma_{\varphi_{b}}}{\Gamma},
$$

where

$$
\begin{aligned}
\gamma_{t} & =g_{\varphi_{a} \varphi_{a}} g_{\varphi_{b} \varphi_{b}}-g_{\varphi_{a} \varphi_{b}}^{2} \\
\gamma_{\varphi_{a}} & =g_{t \varphi_{b}} g_{\varphi_{a} \varphi_{b}}-g_{t \varphi_{a}} g_{\varphi_{b} \varphi_{b}} \\
\gamma_{\varphi_{b}} & =g_{t \varphi_{a}} g_{\varphi_{a} \varphi_{b}}-g_{t \varphi_{b}} g_{\varphi_{a} \varphi_{a}}
\end{aligned}
$$

and the determinant of the time and azimuthal angle components is defined as

$$
\begin{aligned}
\Gamma & =g_{t t} g_{\varphi_{a} \varphi_{a}} g_{\varphi_{b} \varphi_{b}}+2 g_{t \varphi_{a}} g_{t \varphi_{b}} g_{\varphi_{a} \varphi_{b}}-g_{t \varphi_{a}}^{2} g_{\varphi_{b} \varphi_{b}}-g_{t \varphi_{b}}^{2} g_{\varphi_{a} \varphi_{a}}-g_{\varphi_{a} \varphi_{b}}^{2} g_{t t} \\
& =g_{t t} \gamma_{t}+g_{t \varphi_{a}} \gamma_{\varphi_{a}}+g_{t \varphi_{b}} \gamma_{\varphi_{b}},
\end{aligned}
$$

Inserting the five-dimensional Kerr-AdS black hole solution (3.12), the factor $\gamma$ 's (3.10) are expressed:

$$
\begin{aligned}
\gamma_{t} \times & \frac{r^{2} \rho^{2} \Xi_{a}^{2} \Xi_{b}^{2}}{\sin ^{2} \theta \cos ^{2} \theta} \\
= & r^{2} \Delta\left(-A b^{2} \cos ^{2} \theta-B a^{2} \sin ^{2} \theta+\ell^{-2} a^{2} b^{2}\left(A \cos ^{2} \theta+B \sin ^{2} \theta\right)\right) \\
& +\Delta_{\theta} A^{2} B^{2} \\
\gamma_{\varphi_{a}} \times & \frac{r^{2} \rho^{2} \Xi_{a} \Xi_{b}^{2}}{a \sin ^{2} \theta \cos ^{2} \theta} \\
= & r^{2} \Delta\left(-B+\ell^{-2} b^{2}\left(A \cos ^{2} \theta+B \sin ^{2} \theta\right)\right)+\Delta_{\theta} A B^{2}, \\
\gamma_{\varphi_{b}} \times & \frac{r^{2} \rho^{2} \Xi_{a}^{2} \Xi_{b}}{b \sin ^{2} \theta \cos ^{2} \theta} \\
= & r^{2} \Delta\left(-A+\ell^{-2} a^{2}\left(A \cos ^{2} \theta+B \sin ^{2} \theta\right)\right)+\Delta_{\theta} A^{2} B
\end{aligned}
$$

and $\Gamma$ of Eq.(D.3) is expressed:

$$
\Gamma=-\frac{\sin ^{2} \theta \cos ^{2} \theta r^{2} \Delta \Delta_{\theta}}{\Xi_{a}^{2} \Xi_{b}^{2}} .
$$


The contravariant metric of time and azimuthal components are obtained using Eqs.(D.1)-(D.7):

$$
\begin{aligned}
g^{t t}= & \frac{1}{r^{2} \rho^{2} \Delta_{\theta}}\left(A b^{2} \cos ^{2} \theta+B a^{2} \sin ^{2} \theta-\ell^{-2} a^{2} b^{2}\left(A \cos ^{2} \theta+B \sin ^{2} \theta\right)\right) \\
& -\frac{1}{r^{4} \rho^{2} \Delta} A^{2} B^{2}, \\
g^{t \varphi_{a}}= & \frac{a \Xi_{a}}{r^{2} \rho^{2}}\left(\frac{1}{\Delta_{\theta}}\left(B-\ell^{-2} b^{2}\left(A \cos ^{2} \theta+B \sin ^{2} \theta\right)-\frac{1}{r^{2} \Delta} A B^{2}\right),\right. \\
g^{t \varphi_{b}}= & \frac{b \Xi_{b}}{r^{2} \rho^{2}}\left(\frac{1}{\Delta_{\theta}}\left(A-\ell^{-2} a^{2}\left(A \cos ^{2} \theta+B \sin ^{2} \theta\right)-\frac{1}{r^{2} \Delta} A^{2} B\right),\right.
\end{aligned}
$$

which are the expressions (3.14)-(3.16).

\section{References}

[1] For textbooks of general relativity and black holes, see for example, C.W. Misner, K.S. Thorne and J.A. Wheeter, Gravitation, (W.H. Freeman and Company,San Francisco, 1972); R.M. Wald, General Relativity, (The University of Chicago Press, Chicago,1984).

[2] For a review article, see for example, D.K. Townsend, gr-qc/9707012.

[3] For exact solutions, H. Stephani, D. Kramer, M. Maccallum, C. Hoenselaers and E. Herlt, Exact Solutions to Einstein's Field Equation (second Edition), Cambridge University Press (2003).

[4] R.C. Myers and M.J. Perry, Ann. Phys. 172, (1986).

[5] S.W.Hawking, G.J. Hunter and M.M.Taylor-Robinson, Phys. Rev. D59, 064005 (1999).

[6] G.W.Gibbons, H. Lü, D.N. Page and C.N. Pope, J. Geom. Phys., 54, 49 (2004); G.W.Gibbons, M.J. Perry and C.N. Pope, Class. Quantum Grav., 221503 (2005).

[7] Y. Hashimoto, M. Sakaguchi and Y. Yaui, Phys. Lett. B600, 270 (2004); H. KIhara, M. Sakaguchi and Y. Yaui, Phys. Lett. B621, 288 (2005). 
[8] L. Smarr, Phys. Rev. Lett. 30, 71 (1973).

[9] J.D. Bekenstein, Phys. Rev. D7, 2333 (1973); ibid. D9, 3292 (1974).

[10] J.M. Bardeen, B. Carter and S. W. Hawking, Commun. Math. Phys. 31, 161 (1973).

[11] G.W.Gibbons and S.W.Hawking, Phys. Rev. D15, 2752 (1977).

[12] S.W. Hawking, Commun. Math. Phys. 43, 199 (1975).

[13] R. Penrose, Rev. Nouvo Cimento, I, 252 (1969).

[14] D. Cheistdoulou, Phys. Rev. Lett. 25, 1596 (1970); D. Cheistdoulou and R. Ruffini, Phys. Rev. D4, 3552 (1971).

[15] J.B. Hartle and S.W.Hawking, Phys. Rev. D13, 2188 (1976).

[16] G. 't Hooft, Nucl. Phys. B256, 727 (1985).

[17] L. Susskind and J. Uglum, Phys. Rev. D50, 2700 (1994).

[18] S.P. de Alwis and N. Ohta, Phys. Rev. D52, 3529 (1995).

[19] M. Kenmoku, K.K. Nandi and K. Shigemoto, Class. Quant. Grav. 22, 3923 (2005).

[20] K.S. Thorne, W.H. Zurec and R.H. Price, "The Thermal Atmosphere of a Black Hole" in Black Holes: The Membrane Paradigm, edited by K.S. Thorne, R.H.Price and D.A. Macdonald, Yale University Press, (1986)

[21] I. Ichinose and Y. Satoh, Nucl. Phys. B447, 340 (1995).

[22] S-W. Kim, W.T. Kim, Y-J. Park and H. Shin, Phys. Lett. B392, 311 (1997).

[23] J. Ho and G. Kang, Phys. Lett. B445, 27 (1998).

[24] L. Fatibene, M. Ferraris, M. Francaviglia and M. Raiteri, Phys. Rev. D60, 124012 (1999).

[25] M-H. Lee and J.K. Kim, Phys. Rev. D54, 3904 (1996).

[26] R.B. Mann and S.N. Solodukhin, Phys. Rev. D54, 3932 (1996). 
[27] V. Frolov and V. Fursaev, Phys. Rev. D61, 024007 (2000); S. Hod, Phys. Rev. D61, 024018 (2000); B. Wang and E. Abdalla, Phys. Rev. D62, 044030 (2000); J. Jing and M-L. Yan, Phys. Rev. D62, 104013 (2000).

[28] M. Kenmoku, K. Ishimoto, K.K. Nandi and K. Shigemoto, Phys. Rev. D 73, 064004 (2006).

[29] N.D. Birrell and P.C.W. Davis, Quantum Fields in Curved Space, Cambridge University Press (1982).

[30] B. Carter, Commun. Math. Phys. 10, 280 (1968).

[31] B.F. Whiting, J. Math. Phys. 30, 1301 (1989).

[32] S. Mukohyama, Phys. Rev. D 61, 124021 (2000).

[33] V. Cardoso, O.J.C. Dias, J.P.S. Lemos and S. Yoshida, hep-th/040496. 